\title{
A low Fermi scale from a simple gaugino-scalar mass relation
}

\author{
F. Brümmer ${ }^{a, b}$ and W. Buchmüller ${ }^{b}$ \\ ${ }^{a}$ Scuola Internazionale Superiore di Studi Avanzati SISSA/ISAS, \\ Via Bonomea 265, I-34136 Trieste, Italy \\ ${ }^{b}$ Deutsches Elektronen-Synchrotron DESY, \\ Notkestraße 85, D-22607 Hamburg, Germany \\ E-mail: fbruemmer@sissa.it, wilfried.buchmueller@desy.de
}

ABSTRACT: In supersymmetric extensions of the Standard Model, the Fermi scale of electroweak symmetry breaking is determined by the pattern of supersymmetry breaking. We present an example, motivated by a higher-dimensional GUT model, where a particular mass relation between the gauginos, third-generation squarks and Higgs fields of the MSSM leads to a Fermi scale smaller than the soft mass scale. This is in agreement with the measured Higgs boson mass. The $\mu$ parameter is generated independently of supersymmetry breaking, however the $\mu$ problem becomes less acute due to the little hierarchy between the soft mass scale and the Fermi scale as we will argue. The resulting superparticle mass spectra depend on the localization of quark and lepton fields in higher dimensions. In one case, the squarks of the first two generations as well as the gauginos and higgsinos can be in the range of the LHC. Alternatively, only the higgsinos may be accessible at colliders. The lightest superparticle is the gravitino.

Keywords: Supersymmetric Standard Model, Compactification and String Models

ArXiv EPRINT: 1311.1114 


\section{Contents}

1 Introduction 1

2 Electroweak symmetry breaking with a little hierarchy 2

3 Supersymmetry breaking in higher-dimensional GUTs 9

4 Prospects for phenomenology and outlook $\quad 14$

\section{Introduction}

The unification of gauge couplings and the prediction of viable dark matter candidates provides a strong theoretical motivation for supersymmetric extensions of the Standard Model with TeV superparticle masses [1-3]. So far searches for heavy superparticles at the Large Hadron Collider (LHC) have only led to lower bounds on scalar quark and gluino masses of about $1-2 \mathrm{TeV}$ [4-11]. On the other hand, the discovery of a $126 \mathrm{GeV}$ Higgs boson [12-14] allows, without or with supersymmetry, for an extrapolation of the Standard Model up to the scale of grand unification.

The Higgs boson mass is consistent with the mass range predicted by the minimal supersymmetric standard model (MSSM). However, since the Higgs mass significantly exceeds its tree-level upper bound of $91 \mathrm{GeV}$, quantum corrections are large, which generically requires multi-TeV scalar masses. This raises the question why the Fermi scale, the expectation value of the Higgs field, $\langle H\rangle=\left(\sqrt{2} G_{F}\right)^{-1 / 2}=246 \mathrm{GeV}$, is much smaller than the scale of supersymmetry breaking, and the required fine-tuning of seemingly unrelated parameters is often considered as unnatural. Possible answers to this question invoke the anthropic principle and the string landscape, as in split supersymmetry $[15,16]$, the focus point idea [17-20], or similar accidental cancellations between non-universal gaugino and scalar masses at the grand unification scale [21-27]. The naturalness problem might also be solved in a non-minimal extension of the MSSM with additional sub-TeV degrees of freedom (for instance, the NMSSM, reviewed in [28]), or through non-decoupling effects such as in [29]. ${ }^{1}$

In this note we restrict ourselves to the MSSM, and attempt to answer a question which is intimately connected with the naturalness problem: is there a well motivated and simple set of boundary conditions for the GUT-scale soft terms which favours a 'little hierarchy' between the soft and the electroweak scale? And, what can we expect from this soft mass pattern for the upcoming second LHC run?

Our main findings can be summarized as follows. Since the $\mu$ parameter of the MSSM can be generated independently of supersymmetry breaking, it is technically natural to

\footnotetext{
${ }^{1}$ See [30] for a recent review of naturalness in supersymmetry in the light of the first LHC run.
} 
choose it smaller than the typical soft SUSY breaking parameters, say, of the order of the electroweak scale. Usually, explaining why $\mu$ should be of the order of the soft masses is a well known challenge (the ' $\mu$ problem') in SUSY model building. Here, as we will argue, the $\mu$ problem becomes less severe once one accepts a little hierarchy. To obtain proper electroweak symmetry breaking at large $\tan \beta$, the loop-corrected up-type Higgs soft mass needs to be of the same order as $\mu$ at the scale where the MSSM is matched to the Standard Model, requiring an accidental cancellation between the tree-level and radiative contributions to this parameter. We identify a simple soft mass pattern which suggests this cancellation, and which is motivated by a six-dimensional GUT model (although we expect that there are other models that can lead to the same pattern). Within this model, we obtain an estimate for the possible range of the gluino mass, which will be partly probed at LHC-14. Squarks and sleptons may also be within reach, and by construction there are higgsino-like charginos and neutralinos with electroweak-scale masses which can be discovered at a linear collider.

Note that we are not claiming to solve the fine-tuning problem: the fine-tuning in our model is as large as one would expect from a generic MSSM-type model without large contributions to the lightest Higgs mass from stop mixing, i.e. at the permille level. Our model predicts the relevant soft terms only up to factors of order one, and while the predicted pattern non-trivially allows for a little hierarchy, these unknown factors still need to be tuned in order to actually realize it. We anticipate that fully understanding the origin of the cancellations involved will require a better understanding of the complete UV theory.

\section{Electroweak symmetry breaking with a little hierarchy}

Matching the MSSM to the Standard Model. The scalar potential for the MSSM Higgs fields depends on the higgsino mass $\mu$, which is a parameter of the superpotential, and the soft supersymmetry breaking parameters $m_{H_{u}}^{2}, m_{H_{d}}^{2}$ and $B \mu$,

$$
\begin{aligned}
V= & \left(m_{H_{u}}^{2}+|\mu|^{2}\right) H_{u}^{\dagger} H_{u}+\left(m_{H_{d}}^{2}+|\mu|^{2}\right) H_{d}^{\dagger} H_{d}+B \mu\left(H_{u}^{T} i \sigma_{2} H_{d}+\text { c.c. }\right) \\
& +\frac{1}{8}\left(g^{2}+g^{\prime 2}\right)\left(H_{u}^{\dagger} H_{u}-H_{d}^{\dagger} H_{d}\right)^{2}+\frac{1}{2} g^{2} H_{u}^{\dagger} H_{d} H_{d}^{\dagger} H_{u} .
\end{aligned}
$$

Our starting assumption is that the only scalar with an electroweak-scale mass is the lightest Higgs, while all others (in particular the remaining Higgs bosons) are much heavier. In this so-called decoupling limit the Higgs vacuum expectation value (vev) is approximately aligned with the lightest mass eigenstate. It is convenient to work with the fields $H$ and $H^{\prime}$ defined by ${ }^{2}$

$$
H_{u}=\sin \beta H+\cos \beta i \sigma_{2} H^{*}, \quad H_{d}=\cos \beta i \sigma_{2} H^{*}+\sin \beta H^{\prime},
$$

with

$$
\tan 2 \beta=\frac{2 B \mu}{m_{H_{u}}^{2}-m_{H_{d}}^{2}}
$$

\footnotetext{
${ }^{2}$ In the usual notation for the tree-level mass eigenstates, would-be Goldstone bosons, and mixing angles (see e.g. [31]) this corresponds to $H=\left(G^{+}, v+\left(h^{0}+i G^{0}\right) / \sqrt{2}\right)^{T}, H^{\prime}=\left(\left(H^{0}+i A^{0}\right) / \sqrt{2}, H^{+*}\right)^{T}$, and $\alpha=\beta-\pi / 2$.
} 
such that the quadratic part of the potential is diagonal in the new fields:

$$
\begin{aligned}
V= & m^{2} H^{\dagger} H+m^{\prime 2} H^{\prime \dagger} H^{\prime} \\
& +\frac{1}{8}\left(g^{2}+g^{\prime 2}\right)\left(\cos 2 \beta\left(H^{\dagger} H-H^{\prime \dagger} H^{\prime}\right)-\sin 2 \beta\left(H^{T} i \sigma_{2} H^{\prime}+\text { c.c. }\right)\right)^{2} \\
& +\frac{1}{2} g^{2} H^{\dagger} H^{\prime} H^{\prime \dagger} H,
\end{aligned}
$$

where

$$
\begin{aligned}
& m^{2}=|\mu|^{2}+m_{H_{u}}^{2} \sin ^{2} \beta+m_{H_{d}}^{2} \cos ^{2} \beta-B \mu \sin 2 \beta, \\
& m^{2}=|\mu|^{2}+m_{H_{u}}^{2} \cos ^{2} \beta+m_{H_{d}}^{2} \sin ^{2} \beta+B \mu \sin 2 \beta .
\end{aligned}
$$

Within the MSSM the measured mass of the lightest Higgs boson requires large radiative corrections from heavy stop squarks. Therefore we take the scale $M_{S}=\left(m_{\tilde{t}_{1}} m_{\tilde{t}_{2}}\right)^{1 / 2}$ to be much larger than the electroweak scale, of the order of several TeV. At the scale $M_{S}$ the MSSM is matched to the Standard Model with scalar potential

$$
V=m^{2} H^{\dagger} H+\frac{1}{2} \lambda\left(H^{\dagger} H\right)^{2},
$$

where

$$
\left.\lambda\right|_{M_{S}}=\left.\frac{1}{4}\left(g^{2}+g^{\prime 2}\right) \cos ^{2} 2 \beta\right|_{M_{S}} .
$$

The Higgs mass parameter $m^{2}$ encodes the prediction for the electroweak scale, $v^{2}=$ $-m^{2} / \lambda$, with $\lambda$ being $\mathcal{O}(1)$.

Conditions for a little hierarchy. When keeping the electroweak scale fixed, the treelevel contribution to the lightest Higgs mass is maximized at large $\tan \beta$ (since in that limit $|\cos 2 \beta| \rightarrow 1$ in eq. (2.8)), approaching its limit value of $m_{Z}=91 \mathrm{GeV}$. The region of at least moderately large $\tan \beta \gtrsim 10$ is therefore favoured by the large observed Higgs mass of $126 \mathrm{GeV}$, with the discrepancy accounted for by radiative corrections. The Standard Model-like Higgs field $H$ is then predominantly $H_{u}$.

By eq. (2.3), using $\tan 2 \beta=2 /(\cot \beta-\tan \beta)$, large $\tan \beta$ implies

$$
B \mu \ll m_{H_{d}}^{2}
$$

in the generic case that $\left|m_{H_{u}}^{2}\right| \lesssim m_{H_{d}}^{2} \cdot{ }^{3}$ In the following we will take the $\mu$ parameter to be generated independently of supersymmetry breaking. Since $\mu$ and $B \mu$ are both governed by a Peccei-Quinn symmetry, unless $B \mu$ is merely accidentally small due to radiative corrections, the reason underlying relation (2.9) is that the effective symmetry breaking scale is below the soft mass scale, as will be discussed in more detail momentarily. In that case also $\mu$ is small:

$$
|\mu|^{2} \ll m_{H_{d}}^{2} .
$$

\footnotetext{
${ }^{3}$ We do not consider exceptionally small values for $m_{H_{d}}^{2}$, which could occur in exotic mediation schemes or be induced by RG running at large $y_{b}$ (i.e. extremely large $\tan \beta \gtrsim 40$ ). Some more details about the running of $m_{H_{d}}^{2}$ are given below.
} 
Furthermore, since at large $\tan \beta$ we have ${ }^{4}$

$$
m^{2} \simeq|\mu|^{2}+m_{H_{u}}^{2}+\frac{m_{H_{u}}^{2}-m_{H_{d}}^{2}}{\tan ^{2} \beta},
$$

a little hierarchy requires that $m_{H_{u}}^{2}$ is small,

$$
\left|m_{H_{u}}^{2}\right| \ll m_{H_{d}}^{2} .
$$

Together with eq. (2.3) this implies

$$
\tan \beta \simeq \frac{m_{H_{d}}^{2}}{B \mu}
$$

Relations (2.9), (2.10) and (2.12) are thus necessary to obtain a Fermi scale much smaller than the soft mass scale, assuming that $\tan \beta$ is at least moderately large and that $\mu$ and $B \mu$ are connected. We now proceed to discuss the possible origins of these conditions.

Why should $\boldsymbol{m}_{\boldsymbol{H}_{u}}^{2}$ be small? Choosing $\mu$ and $B \mu$ small is technically natural, and this choice is radiatively stable. By contrast, radiative corrections to the Higgs soft masses are sizeable. In particular, as is well known, no symmetry protects $m_{H_{u}}^{2}$ from loop corrections due to the large top Yukawa coupling. Condition (2.12) is technically unnatural, which is a manifestation of the usual fine-tuning problem in the MSSM. It requires large cancellations between the radiative contributions and the tree-level value of $m_{H_{u}}^{2}$. Let us discuss these in some more detail.

When considering models whose fundamental parameters are defined at the GUT scale, then the Higgs potential will receive large logarithmically enhanced quantum corrections, which need to be resummed using the MSSM renormalization group equations. In addition, there are finite corrections at the matching scale $M_{S}$ which we cannot neglect.

Turning first to the renormalization group running, the tree-level RG-improved Higgs potential at $M_{S}$ can be expressed as a function of the running Higgs mass parameters and of the running gauge couplings. The Higgs mass parameters at the scale $M_{S}$ depend on their GUT-scale values, but also on the GUT-scale soft masses of all fields with sizeable couplings to the Higgs sector. These are the third-generation scalars and the gauginos (with the gluino entering because of its large coupling to the stops and sbottoms). We find for $\tan \beta=15$

$$
\begin{aligned}
\left.m_{H_{u}}^{2}\right|_{M_{S}}= & -\left\{\begin{array}{l}
1.09 \\
1.13 \\
1.18
\end{array}\right\} \widehat{M}_{3}^{2}-\left\{\begin{array}{l}
0.10 \\
0.11 \\
0.11
\end{array}\right\} \widehat{M}_{3} \widehat{M}_{2}+0.22 \widehat{M}_{2}^{2}+0.26 \widehat{M}_{3} \widehat{A}_{t}+0.07 \widehat{M}_{2} \widehat{A}_{t} \\
& -0.12 \widehat{A}_{t}^{2}+\left\{\begin{array}{l}
0.67 \\
0.67 \\
0.66
\end{array}\right\} \widehat{m}_{H_{u}}^{2}-0.24 \widehat{m}_{U_{3}}^{2}-\left\{\begin{array}{l}
0.33 \\
0.33 \\
0.34
\end{array}\right\} \widehat{m}_{Q_{3}}^{2}, \\
\text { for } M_{S}= & \left\{\begin{array}{c}
6.5 \\
5 \\
3.5
\end{array}\right\} \mathrm{TeV} .
\end{aligned}
$$

\footnotetext{
${ }^{4}$ The last term in eq. (2.11) is often neglected. However, in the case of a large matching scale $M_{S}$ it is generally important, even for large values of $\tan \beta$.
} 
Here the hatted quantities on the r.h.s. denote GUT-scale soft parameters, with $\widehat{A}_{t}$ normalized to the top Yukawa coupling. We have taken the GUT scale to be fixed at $M_{\mathrm{GUT}}=1.5 \times 10^{16} \mathrm{GeV}$, and omitted all terms with coefficients smaller than 0.05 . The coefficients are largely insensitive to $\tan \beta$, as long as $\tan \beta \gtrsim 10$; for instance, for $\tan \beta=30$ the coefficients of the $\widehat{M}_{3}^{2}$ term are $-\{1.07 ; 1.11 ; 1.16\}$ and all other coefficients differ from eq. (2.14) at most by 0.01 . Another source of uncertainty is the experimental uncertainty in the top mass. We have checked that the uncertainty obtained from varying $m_{t}$ by $1 \sigma$ around its central value of $173.2 \mathrm{GeV}$ is of similar order, changing the coefficients at most by 0.01 . The GUT-scale values for Yukawa and gauge couplings have been obtained using the two-loop RG code SOFTSUSY [32].

With the assumptions of large $\tan \beta$ and of negligible stop mixing at the GUT scale, i.e. negligible $\widehat{A}_{t}$, the matching scale is in principle rather sharply determined by the lightest Higgs mass $m_{h^{0}}$. This is because the radiative corrections to $m_{h^{0}}$ depend mainly on the stop masses (and on the RG-induced stop mixing parameter at the $\mathrm{TeV}$ scale). In practice however there is still a large uncertainty, partly because of the uncertainty in $y_{t}$, but mostly because of the theory uncertainty in computing $m_{h^{0}}$ from a given soft mass spectrum. We have chosen $M_{S}=5 \pm 1.5 \mathrm{TeV}$, which is in good accordance with the two-loop spectrum codes SOFTSUSY, SuSpect [33] and FeynHiggs [34-37] and also compatible with the threeloop analysis in [38] which is based on the H3M code [39].

The equivalent of eq. (2.14) for $m_{H_{d}}^{2}$ reads

$$
\begin{aligned}
\left.m_{H_{d}}^{2}\right|_{M_{S}} & =-\left\{\begin{array}{l}
0.06 \\
0.07 \\
0.07
\end{array}\right\} \widehat{M}_{3}^{2}+\left\{\begin{array}{l}
0.37 \\
0.38 \\
0.38
\end{array}\right\} \widehat{M}_{2}^{2}+\left\{\begin{array}{l}
0.95 \\
0.95 \\
0.94
\end{array}\right\} \widehat{m}_{H_{d}}^{2}-0.06 \widehat{m}_{U_{3}}^{2}, \\
\text { for } M_{S} & =\left\{\begin{array}{c}
6.5 \\
5 \\
3.5
\end{array}\right\} \mathrm{TeV} .
\end{aligned}
$$

While the coefficients in eq. (2.15) show a more pronounced $\tan \beta$-dependence, the overall running of $m_{H_{d}}^{2}$ remains moderate in the range $10 \lesssim \tan \beta \lesssim 40$. In this region $\left.m_{H_{d}}\right|_{M_{S}}$ is therefore of the order of $\widehat{m}_{H_{d}}$, which is generically of the order $M_{S}$.

In addition to the RG running of the tree-level parameters there are important finite corrections to the Higgs potential due to top-stop loops, which affect the Higgs masses (for a detailed discussion and references, see e.g. [40]). They amount to replacing $m_{H_{u, d}}^{2}$ in eqs. (2.9)-(2.13) by $\bar{m}_{H_{u, d}}^{2}$, where

$$
\bar{m}_{H_{u}}^{2}=m_{H_{u}}^{2}\left(M_{S}\right)-\frac{t_{2}}{v \sin \beta}, \quad \bar{m}_{H_{d}}^{2}=m_{H_{d}}^{2}\left(M_{S}\right)-\frac{t_{1}}{v \cos \beta} .
$$

Here the tadpole terms $t_{i}$ are computed from the minimization conditions for the full one-loop effective potential [40]. Using the one-loop results of [40], it turns out that the dominant corrections to the Higgs masses are obtained in the limit where the top Yukawa coupling is the only non-vanishing coupling, and where the stop squarks are approximately unmixed and degenerate with mass $m_{\tilde{t}}=M_{S}$. In the $\overline{\mathrm{MS}}$ scheme at the renormalization 
scale $M_{S}$, one finds

$$
t_{1} \approx 0, \quad \frac{t_{2}}{v \sin \beta} \approx \frac{3 y_{t}^{2}}{8 \pi^{2}} m_{\tilde{t}}^{2},
$$

i.e., only $m_{H_{u}}^{2}$ is significantly modified by the finite corrections to the Higgs potential.

In this paper we are interested in the question how electroweak symmetry breaking can occur at a scale significantly below the scale of supersymmetry breaking, i.e. how the conditions $m^{2}<0$ and $\left|m^{2}\right| \ll M_{S}^{2}$ can be realized from eq. (2.11) at small $|\mu|$. In particular, how can relation (2.12) be satisfied? An important observation is that in eq. (2.14) the scalar contributions approximately cancel for equal stop and $H_{u}$ masses, $\widehat{m}_{H_{u}}^{2}=\widehat{m}_{Q_{3}}^{2}=\widehat{m}_{U_{3}}^{2} \equiv m_{0}^{2}$. This is the basis of the 'focus point' idea [17-20]. However, as the matching scale increases, the cancellation between the scalar soft mass contributions becomes less precise. The actual focussing point of the RG trajectories, where the $m_{0}$ coefficient vanishes, is only obtained for $M_{S}$ close to the electroweak scale.

As eq. (2.14) shows, the remaining positive contribution to $m_{H_{u}}^{2}$ by scalar masses can be compensated by the negative contribution from gaugino masses. Assuming universal gaugino masses as suggested by unification, $\widehat{M}_{3}=\widehat{M}_{2}=\widehat{M}_{1}=M_{1 / 2}$, and taking the correction eq. (2.17) into account, one obtains for $M_{S}=5 \mathrm{TeV}$

$$
\left.\bar{m}_{H_{u}}^{2}\right|_{M_{S}}=-1.08 M_{1 / 2}^{2}+0.33 M_{1 / 2} \widehat{A}_{t}-0.12 \widehat{A}_{t}^{2}+0.08 m_{0}^{2},
$$

subject to the uncertainties mentioned above. In the following we shall be interested in the case $\left|\widehat{A}_{t}\right| \lesssim M_{1 / 2}$. A cancellation between the gaugino and the scalar contribution then occurs for a particular ratio $M_{1 / 2} / m_{0}$ :

$$
M_{1 / 2}=\kappa m_{0}, \quad \frac{1}{5} \lesssim \kappa \lesssim \frac{1}{3} .
$$

This can also be seen from figure 1, which shows $\bar{m}_{H_{u}}^{2}\left(M_{S}\right)$ as a function of $M_{S}$ for different values of the ratio $\kappa=M_{1 / 2} / m_{0}$ at negative, vanishing, and positive $\widehat{A}_{t}$.

Models predicting a relation of this type therefore show some promise for obtaining a little hierarchy. In section 3 we will present an example with all the required properties: a moderate suppression for the gaugino masses and trilinear terms of roughly the correct size, and a good motivation for near-universal GUT-scale soft masses of the third generation squarks and Higgs fields. For now we still need to justify a remaining key assumption, namely that of small $\mu$ and small $B \mu$.

Why should $\mu$ and $B \mu$ be small? It is well known that the higgsino mass $\mu$ plays a special role among the dimensionful parameters of the MSSM. It preserves supersymmetry, but it breaks a U(1) Peccei-Quinn (PQ) symmetry under which the Higgs bilinear is charged. The soft masses and trilinear soft terms, by contrast, break supersymmetry but preserve $\mathrm{U}(1)_{\mathrm{PQ}}$. The Higgs soft mass mixing parameter $B \mu$ breaks both SUSY and PQ symmetry.

For concreteness, assume that SUSY is broken by some singlet spurion $X$ with $\langle X\rangle=$ $F_{X} \theta^{2}$, and that $\mathrm{U}(1)_{\mathrm{PQ}}$ is broken supersymmetrically by some spurion $Y$, such that the 


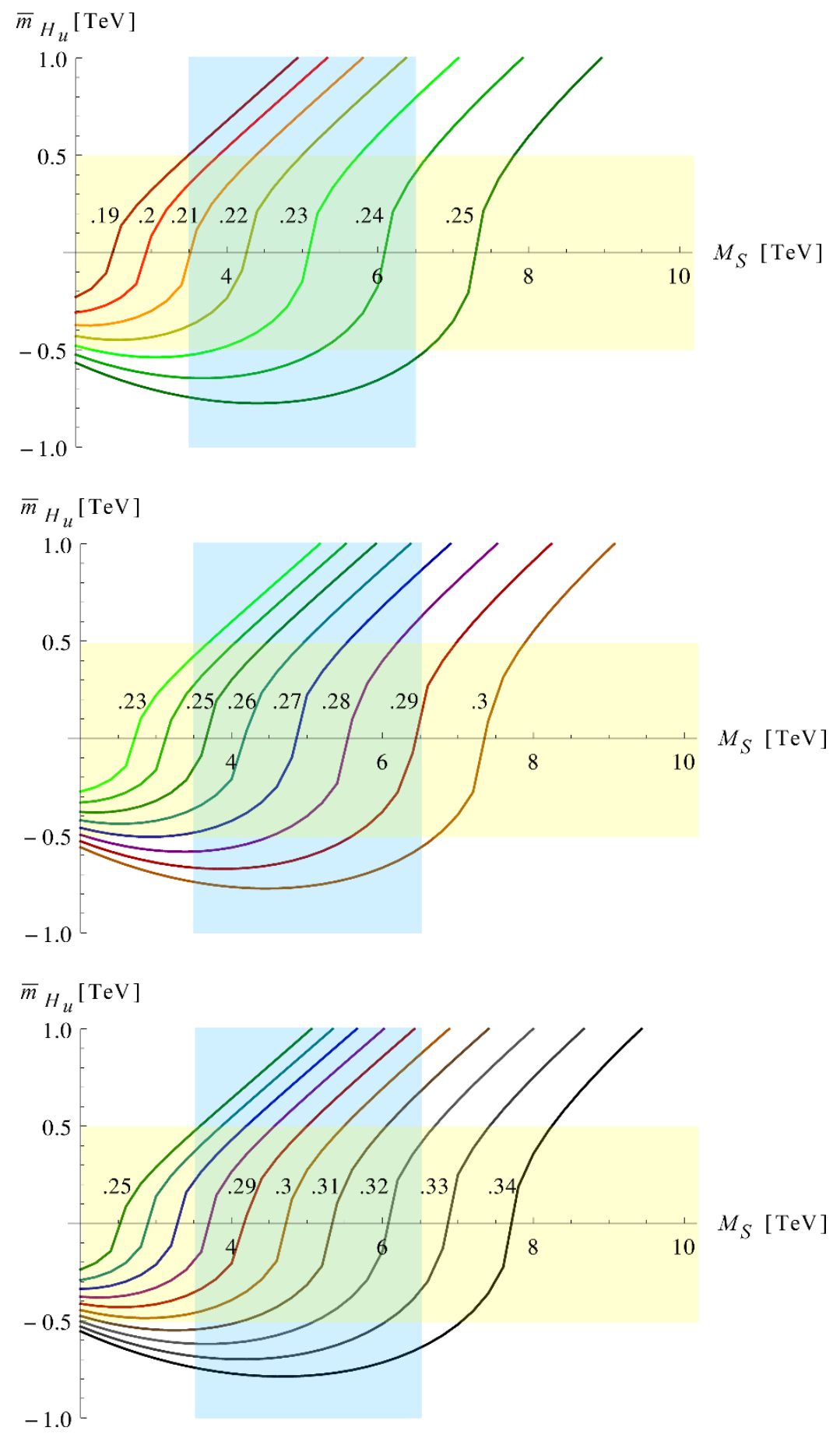

Figure 1. $\bar{m}_{H_{u}}$ (more precisely $\bar{m}_{H_{u}}^{2} / \sqrt{\left|\bar{m}_{H_{u}}^{2}\right|}$ ) as a function of the matching scale $M_{S}$ for various values of the parameter $\kappa=M_{1 / 2} / m_{0}$. Top: $\widehat{A}_{t}=-M_{1 / 2}$, center: $\widehat{A}_{t}=0$, bottom: $\widehat{A}_{t}=+M_{1 / 2}$. Here $\tan \beta=15$ and $M_{\mathrm{GUT}}=1.5 \times 10^{16} \mathrm{GeV}$. We have indicated the range of $M_{S}$ preferred by the Higgs mass (which we took to be $5 \pm 1.5 \mathrm{TeV}$ ) in blue, and a range of $\left|\bar{m}_{H_{u}}\right|$ around the electroweak scale in yellow. 
following terms are allowed in the Lagrangian:

$$
\mathcal{L}=\int d^{2} \theta \frac{Y^{p}}{M^{p-1}}\left(1+\frac{X}{M}\right) H_{u} H_{d}+\int d^{4} \theta \frac{X}{M}\left(\left|H_{u}\right|^{2}+\left|H_{d}\right|^{2}\right)+\text { h.c. }
$$

The Kähler terms in eq. (2.20) can be absorbed in the superpotential terms by a field redefinition. The power $p$ depends on the PQ charges of $H_{u} H_{d}$ and of $Y$. Bare $\mu$ and $B \mu$ terms $\left.\mu H_{u} H_{d}\right|_{\theta^{2}}$ and $\left.X H_{u} H_{d}\right|_{\theta^{2}}$ are forbidden by $\mathrm{U}(1)_{\mathrm{PQ}}$, which also forbids the operators $\left.X^{\dagger} H_{u} H_{d}\right|_{\theta^{2} \bar{\theta}^{2}}$ and $\left.|X|^{2} H_{u} H_{d}\right|_{\theta^{2} \bar{\theta}^{2}}$. Consequently, the effective $\mu$ parameter is

$$
\mu \sim \frac{Y^{p}}{M^{p-1}}
$$

and $B \mu$ is proportional to both $\mu$ and the SUSY-breaking vev,

$$
B \mu \sim \frac{Y^{p}}{M^{p}} F_{X} \sim \mu M_{S}
$$

where $F_{X} / M \sim M_{S}$ is the scale of the scalar and gaugino soft mass parameters.

Choosing $Y$ such that

$$
|\mu|^{2} \ll M_{S}^{2}
$$

is technically natural, since PQ breaking is a priori unrelated to SUSY breaking. The ' $\mu$ problem' is usually formulated as the need for an explanation why the SUSY-breaking soft masses are of the same order as $\mu$. Here this is not the case: in contrast to the common SUSY model building approach, we obtain $\mu$ and the SUSY breaking soft terms from two independent scales. As soon as we allow for a little hierarchy, the $\mu$ problem becomes less severe as we will argue momentarily. Indeed the most interesting parameter choice has $\mu$ maximally separated from $M_{S}$, to the extent that is allowed by experimental data.

With the conditions (2.12) and (2.23), electroweak symmetry can be broken with all three terms in eq. (2.11) being of the order of the electroweak scale. The required finetuning is no worse than the fine-tuning needed in the more common case where $\mu$ is of the order of the soft breaking terms, and cancelled against a similarly large $\bar{m}_{H_{u}}^{2}$. In our case we are instead cancelling large radiative contributions to the $\bar{m}_{H_{u}}^{2}$ parameter against each other.

Remarkably, if the conditions (2.23) are satisfied with $\bar{m}_{H_{u}}^{2}$ sufficiently small, then the electroweak scale is parametrically given not by $M_{S}$ but by $\mu$. This is most easily seen by setting $\bar{m}_{H_{u}}^{2}=0, m_{H_{d}}=\eta M_{S}, B \mu=\zeta|\mu| M_{S}$ at the scale $M_{S}$, with $\eta$ and $\zeta$ of the order one (or at least small compared to $M_{S} / \mu$ - in the next section we will consider a model where $\zeta \sim 1 / \kappa$, with $\kappa \approx 0.25$ as in eq. (2.19)). One then obtains

$$
m_{H}^{2}=\left(\begin{array}{cc}
|\mu|^{2} & \zeta|\mu| M_{S} \\
\zeta|\mu| M_{S} & \eta^{2} M_{S}^{2}
\end{array}\right),
$$

leading to

$$
-m^{2} \simeq\left(\frac{\zeta^{2}}{\eta^{2}}-1\right)|\mu|^{2}
$$


For $\zeta^{2}>\eta^{2}$ the Higgs mass matrix eq. (2.24) has a negative eigenvalue even though the diagonal entries are both positive. In fact, for $\zeta^{2} \gg \eta^{2}$ the electroweak scale is given by a seesaw-type formula,

$$
m^{2} \simeq-\frac{\left(\zeta|\mu| M_{S}\right)^{2}}{\eta^{2} M_{S}^{2}}=-\frac{\zeta^{2}}{\eta^{2}}|\mu|^{2}<0 .
$$

A very similar pattern has previously been investigated in the context of gauge-mediated supersymmetry breaking, where the hierarchy between $m_{H_{d}}$ (or equivalently $M_{S}$ ) and $|\mu|$ is not due to a PQ symmetry but due to a loop factor [41, 42]. Let us emphasize that a sufficiently large value of $B \mu$, and therefore $\zeta$, is crucial for electroweak symmetry breaking, which takes place irrespective of the sign of $m_{H_{u}}^{2}$.

As already emphasized we have no symmetry reason for $\bar{m}_{H_{u}}^{2}=0$. In the more general case $\left|\bar{m}_{H_{u}}^{2}\right| \ll M_{S}^{2}$, electroweak symmetry breaking imposes a lower bound on $|\mu|^{2}$,

$$
|\mu|^{2}>\frac{\eta^{2}}{\zeta^{2}-\eta^{2}} \bar{m}_{H_{u}}^{2}
$$

Note that there is also a phenomenological lower bound on $|\mu|$ : $\operatorname{since} \tan \beta$ is parametrically given by $m_{H_{d}}^{2} / B \mu \sim \eta^{2} M_{S} /(\zeta|\mu|)$, and should not exceed a value $\approx 60$ in order to avoid non-perturbative Yukawa couplings, the hierarchy between $\mu$ and $M_{S}$ cannot be too large. Thus, for fixed $M_{S}, \mu$ is bounded from below. The most relevant bound for the model of the next section will however turn out to be the direct experimental lower limit $|\mu| \gtrsim 100 \mathrm{GeV}$ from chargino searches at LEP.

At this point let us briefly return to the $\mu$ problem. If we set $\bar{m}_{H_{u}}^{2}=0$ and ignore the associated fine-tuning for a moment, it is clear from the Higgs mass matrix eq. (2.24) and from eq. (2.26) that the soft mass scale may be decoupled from the scale of electroweak symmetry breaking (which is essentially given by $\mu$ ). In a hypothetical universe with very light down-type quarks, there would also be no restriction on the ratio $M_{S} / \mu \sim \tan \beta$, so $M_{S}$ could in principle be very large, and the $\mu$ problem would be circumvented. Realistically, however, this line of reasoning is invalidated to some extent by the experimentally known bottom and top quark masses. The known value of $m_{b}$ leads to an upper bound on $\tan \beta$, while the known value of $m_{t}$ implies that the top Yukawa coupling is large, and that a relation such as $\bar{m}_{H_{u}}^{2}=0$ will therefore be spoiled by large loop corrections. These two arguments point towards a soft mass scale $M_{S}$ which is not too far above the electroweak scale; the $126 \mathrm{GeV}$ Higgs mass further fixes the 'little hierarchy' to amount to 1-2 decades. In summary, the $\mu$ problem is still present, but somewhat alleviated when allowing for a little hierarchy between $M_{S}$ and the Fermi scale (as seems to be forced upon us by LHC data).

\section{Supersymmetry breaking in higher-dimensional GUTs}

We shall now present an explicit example which realizes the conditions for a seesaw-type pattern of electroweak symmetry breaking discussed in the previous section. Consider a six-dimensional (6d) GUT model, with the third quark-lepton generation and the Higgs fields located in the bulk and the first two families localized at $4 \mathrm{~d}$ branes or orbifold fixed 
points. Such a model has been derived as an intermediate step [43] in a compactification of the heterotic string to the supersymmetric standard model in four dimensions [44, 45]. Supersymmetry is supposed to be broken by the $F$-term of a chiral superfield located at some fixed point.

In the following we shall restrict our discussion to the case of strong coupling at the cutoff scale. The couplings of the supersymmetry breaking brane field to Higgs, matter and gauge fields can then be estimated by means of 'naive dimensional analysis' (NDA) following [51]. The localization of the fields fixes the structure of the Lagrangian

$$
\mathcal{L}_{6 d}=\mathcal{L}_{\text {bulk }}\left(W_{\alpha}, \Phi\right)+\sum_{i} \delta^{2}\left(y-y_{i}\right) \mathcal{L}_{i}\left(W_{\alpha}, \Phi, \phi\right)
$$

where $y_{i}$ are the positions of the $4 \mathrm{~d}$ branes, and $W_{\alpha}, \Phi$ and $\phi$ denote bulk gauge fields, bulk chiral fields, and brane chiral fields, respectively. Matching $6 \mathrm{~d}$ and $4 \mathrm{~d}$ theories at the compactification scale, the gauge couplings and Planck masses are related by

$$
\frac{1}{g_{6}^{2}} V_{2}=\frac{1}{g_{4}^{2}}, \quad M_{6}^{4} V_{2}=M_{4}^{2},
$$

where $V_{2}$ is the volume of the two compact dimensions. ${ }^{5}$

In order to define the theory one has to introduce a UV cutoff $\Lambda$. If loop corrections at the scale $\Lambda$ are suppressed by $\epsilon$, the Lagrangian eq. (3.1) can be expressed in terms of dimensionless fields $\widehat{W}_{\alpha} / \Lambda^{3 / 2}, \widehat{\Phi} / \Lambda$ and $\widehat{\phi} / \Lambda$,

$$
\begin{aligned}
W_{\alpha}(x, y) & =\frac{\Lambda^{5 / 2}}{\left(\epsilon \ell_{6}\right)^{1 / 2}} \frac{\widehat{W}_{\alpha}(x, y)}{\Lambda^{3 / 2}}, \quad \Phi(x, y)=\left(\frac{\Lambda^{4}}{\epsilon \ell_{6}}\right)^{1 / 2} \frac{\widehat{\Phi}(x, y)}{\Lambda}, \\
\phi_{i}(x) & =\left(\frac{\Lambda^{2}}{\epsilon \ell_{4}}\right)^{1 / 2} \frac{\widehat{\phi}_{i}(x)}{\Lambda} .
\end{aligned}
$$

The fields $W_{\alpha}, \Phi$ and $\phi$ are assumed to have canonical kinetic terms in $6 \mathrm{~d}$ and $4 \mathrm{~d}$, respectively, and the rescaled fields $\widehat{W}_{\alpha}, \widehat{\Phi}$ and $\widehat{\phi}$ have canonical dimensions in $4 \mathrm{~d}$. According to NDA the Lagrangian (3.1) now takes the form

$$
\mathcal{L}_{6 d}=\frac{\Lambda^{6}}{\epsilon \ell_{6}} \widehat{\mathcal{L}}_{\text {bulk }}\left(\frac{\widehat{W}_{\alpha}}{\Lambda^{3 / 2}}, \frac{\widehat{\Phi}}{\Lambda}, \frac{\partial}{\Lambda}\right)+\sum_{i} \delta^{2}\left(y-y_{i}\right) \frac{\Lambda^{4}}{\epsilon \ell_{4}} \widehat{\mathcal{L}}_{i}\left(\frac{\widehat{W}_{\alpha}}{\Lambda^{3 / 2}}, \frac{\widehat{\Phi}}{\Lambda}, \frac{\widehat{\phi}}{\Lambda}, \frac{\partial}{\Lambda}\right),
$$

where all couplings are $\mathcal{O}(1)$ and $\ell_{D}=2^{D} \pi^{D / 2} \Gamma(D / 2)$ is a geometrical loop factor, with

$$
\ell_{6}=128 \pi^{3}, \quad \ell_{4}=16 \pi^{2} .
$$

Strong coupling at the cutoff scale $\Lambda$ corresponds to $\epsilon \simeq 1$.

\footnotetext{
${ }^{5}$ In the considered GUT model one has $V_{2}=2 \pi^{2} R_{5} R_{6}$, where $R_{5}$ and $R_{6}$ are the radii of the orbifold. The model has a Wilson line in the direction of $R_{6}$ which breaks the GUT symmetry. With $R_{5} \geq R_{6}$, the mass of the lowest lying Kaluza-Klein state is $1 /\left(2 R_{5}\right)$. Identifying this mass with the GUT scale $M_{\mathrm{GUT}} \simeq 1 \times 10^{16} \mathrm{GeV}$, one obtains $V_{2}^{-1 / 2} \simeq 5 \times 10^{15} \mathrm{GeV}$ (see [46]).
} 
In our 6d GUT model, the couplings of the SUSY breaking brane field $X$ to the bulk fields are given $b y^{6}$

$$
\begin{aligned}
-\mathcal{L}_{s b}=\frac{\Lambda^{4}}{\epsilon \ell_{4}} & \left\{\int \frac{d^{2} \theta}{\Lambda}\left(\frac{\widehat{\mu}}{\Lambda} \frac{\widehat{H}_{u}}{\Lambda} \frac{\widehat{H}_{d}}{\Lambda}\left(1+\frac{\widehat{X}}{\Lambda}\right)+\frac{\widehat{X}}{\Lambda}\left(\operatorname{tr}\left[\frac{\widehat{W}^{\alpha}}{\Lambda^{3 / 2}} \frac{\widehat{W}_{\alpha}}{\Lambda^{3 / 2}}\right]+\frac{\widehat{Q}_{3}}{\Lambda} \frac{\widehat{H}_{u}}{\Lambda} \frac{\widehat{U}_{3}}{\Lambda}\right)+\text { h.c. }\right)\right. \\
& \left.+\int \frac{d^{4} \theta}{\Lambda^{2}} \frac{|\widehat{X}|^{2}}{\Lambda^{2}}\left(\frac{\left|\widehat{H}_{u}\right|^{2}}{\Lambda^{2}}+\frac{\left|\widehat{H}_{d}\right|^{2}}{\Lambda^{2}}+\frac{\left|\widehat{Q}_{3}\right|^{2}}{\Lambda^{2}}+\frac{\left|\widehat{U}_{3}\right|^{2}}{\Lambda^{2}}+\frac{\left|\widehat{D}_{3}\right|^{2}}{\Lambda^{2}}+\frac{\left|\widehat{L}_{3}\right|^{2}}{\Lambda^{2}}+\frac{\left|\widehat{E}_{3}\right|^{2}}{\Lambda^{2}}\right)\right\},
\end{aligned}
$$

where $H_{u}, H_{d}, W_{\alpha}, Q_{3}, U_{3}, D_{3}, L_{3}$ and $E_{3}$ denote Higgs fields, gauge fields and third generation quark and lepton fields, respectively. $H_{u}$ is part of the $6 \mathrm{~d}$ gauge multiplet, $Q_{3}$ and $U_{3}$ belong to the same hypermultiplet, and the cubic term $Q_{3} H_{u} U_{3}$ is part of the $6 \mathrm{~d}$ gauge interactions. ${ }^{7}$ From the gauge kinetic term one reads off the gauge coupling

$$
g_{6} \sim \frac{\left(\epsilon \ell_{6}\right)^{1 / 2}}{\Lambda}
$$

The mass parameter $\widehat{\mu}$ is an additional free parameter which can be much smaller than the cutoff scale $\Lambda$ due to an accidental PQ symmetry as discussed in section 2. From eqs. (3.3) and (3.7) one obtains the Lagrangian for canonically normalized bulk fields,

$$
\begin{aligned}
-\mathcal{L}_{s b}=\frac{\ell_{6}}{\ell_{4}} \frac{1}{\Lambda^{D-4}} & \left\{\int d^{2} \theta\left(\hat{\mu} H_{u} H_{d}\left(1+\frac{\widehat{X}}{\Lambda}\right)+\frac{\widehat{X}}{\Lambda}\left(\operatorname{tr}\left[W^{\alpha} W_{\alpha}\right]+g_{6} Q_{3} H_{u} U_{3}+\text { h.c. }\right)\right)\right. \\
& \left.+\int d^{4} \theta \frac{|\widehat{X}|^{2}}{\Lambda^{2}}\left(\left|H_{u}\right|^{2}+\left|H_{d}\right|^{2}+\left|Q_{3}\right|^{2}+\left|U_{3}\right|^{2}+\left|D_{3}\right|^{2}+\left|L_{3}\right|^{2}+\left|E_{3}\right|^{2}\right)\right\} .
\end{aligned}
$$

Finally, the replacement $\Phi(x, y) \rightarrow V_{2}^{-1 / 2} \Phi(x)$ yields the couplings of canonically normalized zero modes,

$$
\begin{aligned}
&-\mathcal{L}_{s b}=\frac{\ell_{6}}{\ell_{4}} \frac{1}{\Lambda^{2} V_{2}}\left\{\int d^{2} \theta\left(\hat{\mu} H_{u} H_{d}\left(1+\frac{\widehat{X}}{\Lambda}\right)+\frac{\widehat{X}}{\Lambda}\left(\operatorname{tr}\left[W^{\alpha} W_{\alpha}\right]+g_{4} Q_{3} H_{u} U_{3}+\text { h.c. }\right)\right)\right. \\
&\left.+\int d^{4} \theta \frac{|\widehat{X}|^{2}}{\Lambda^{2}}\left(\left|H_{u}\right|^{2}+\left|H_{d}\right|^{2}+\left|Q_{3}\right|^{2}+\left|U_{3}\right|^{2}+\left|D_{3}\right|^{2}+\left|L_{3}\right|^{2}+\left|E_{3}\right|^{2}\right)\right\} .
\end{aligned}
$$

In eq. (3.7) we have assumed a universal coupling of the SUSY breaking field to bulk fields. The focus point cancellation discussed in section 2 requires approximately equal mass terms of $H_{u}, Q_{3}$ and $U_{3}$ at a level of about $5 \%$. In the considered model the equality of mass terms is guaranteed by a symmetry only for $U_{3}$ and $E_{3}$, which belong to the same

\footnotetext{
${ }^{6}$ This model has two pairs of equivalent fixed points [43]. Hence, there will be at least two SUSY breaking fields, at a pair of equivalent fixed points. For the following discussion this complication is irrelevant and will be ignored.

${ }^{7}$ Trilinear terms for the other matter fields are also allowed but will not be written explicitly.
} 
$\mathrm{SU}(6)$ hypermultiplet in six dimensions. For all other fields a dynamical reason is needed. The couplings of brane and bulk fields depend on the profile that the bulk fields aquire in connection with the stabilization of the compact dimensions. These profiles depend on the presence of localized Fayet-Iliopoulos (FI) terms [47]. Such FI-terms are also crucial to reconcile the tree-level gauge-top unification of the model, $y_{t}=g_{4}$ (cf. eq. (3.10)), with the large values of $\tan \beta$ considered in section 2 [46]. It is conceivable that the FI terms present in the model [43] lead to approximately equal mass terms, but a detailed study of the compactification dynamics is beyond the scope of this paper.

Replacing now the brane field $X$ by its SUSY breaking vacuum expectation value $F_{X}$, we obtain from eq. (3.10) the wanted mass parameters of the zero modes for gaugino fields, Higgs and higgsino fields and third generation scalar quark-lepton fields,

$$
\begin{aligned}
\mathcal{L}_{\text {soft }}= & -\left(\frac{1}{2} \mu h_{u} h_{d}+B \mu H_{u} H_{d}+\frac{1}{2} M_{1 / 2} \operatorname{tr} \lambda^{a} \lambda^{a}+A_{t} y_{t} Q_{3} H_{u} U_{3}+\text { h.c. }\right) \\
& -\left(m_{0}^{2}+|\mu|^{2}\right)\left(\left|H_{u}\right|^{2}+\left|H_{d}\right|^{2}\right)-m_{0}^{2}\left(\left|Q_{3}\right|^{2}+\left|U_{3}\right|^{2}+\left|D_{3}\right|^{2}+\left|L_{3}\right|^{2}+\left|E_{3}\right|^{2}\right)
\end{aligned}
$$

where $y_{t}=g_{4}$, and

$$
\begin{aligned}
& m_{0}^{2} \sim \kappa^{2}\left(\frac{\widehat{F}_{X}}{\Lambda}\right)^{2}, \quad M_{1 / 2} \sim \kappa^{2} \frac{\widehat{F}_{X}}{\Lambda} \sim \kappa m_{0}, \quad A_{t} \sim M_{1 / 2} \\
& \mu \sim \kappa^{2} \hat{\mu}, \quad B \mu \sim \kappa^{2} \hat{\mu} \frac{\widehat{F}_{X}}{\Lambda} \sim \frac{1}{\kappa} \mu m_{0},
\end{aligned}
$$

with

$$
\kappa^{2}=\frac{\ell_{6}}{\ell_{4}} \frac{1}{\Lambda^{2} V_{2}}=\frac{\ell_{6}}{\ell_{4}}\left(\frac{M_{6}}{\Lambda}\right)^{2} \frac{1}{M_{4} V_{2}^{1 / 2}}
$$

For $\Lambda=M_{6}$ and GUT-scale extra dimensions, i.e. $V_{2}^{-1 / 2} \simeq 5 \times 10^{15} \mathrm{GeV}$, this yields $\kappa^{2} \simeq 0.06$. For the particularly interesting gaugino-scalar mass relation we then obtain

$$
M_{1 / 2} \sim 0.25 m_{0}
$$

Let us emphasize that this relation is not at all generic, but based on a 6d GUT picture, supersymmetry breaking by a brane field and the assumption of strong coupling at the UV cutoff which is chosen to be the $6 \mathrm{~d}$ Planck mass. The prediction for analogous models with a different number of GUT-scale extra dimensions is not too different, however: the general expression for $D$ dimensions and $\Lambda=M_{D}$ reads

$$
\kappa^{2}=\frac{\ell_{D}}{\ell_{4}}\left(\frac{1}{M_{4}^{D-4} V_{D-4}}\right)^{\frac{2}{D-2}}
$$

and yields $\kappa^{2}=0.08(0.09)$ for $D=5(D=7)$, assuming the same compactification radius as above. For even larger $D$ the loop factor enhancement becomes dominant, and $\kappa^{2}$ grows rather large. The precise choice of the compactification scale sensitively affects 
the prediction for $\kappa^{2}$, and its proper value is dependent on the model details. The following discussion applies to our $D=6$ orbifold model with $V_{2}^{-1 / 2} \simeq 5 \times 10^{15} \mathrm{GeV}$.

Comparing with the electroweak symmetry breaking pattern of the last section, we find that in this model the $\kappa$ parameter is of the correct order of magnitude to explain a small $\bar{m}_{H_{u}}^{2}$ and a little hierarchy between the electroweak scale and the soft mass scale. The $\zeta$ parameter is somewhat large at $\zeta \sim 1 / \kappa \sim 4$; therefore if $\bar{m}_{H_{u}}^{2}$ were completely negligible, we would obtain a slightly too large electroweak scale from eq. (2.26):

$$
v^{2}=-\frac{m^{2}}{\lambda} \approx \frac{(4 \mu)^{2}}{\lambda}
$$

which is not compatible with the experimental lower bound $|\mu| \gtrsim 100 \mathrm{GeV}$. However, a finite negative $\bar{m}_{H_{u}}^{2}$ can easily cure this.

An important quantity is the gravitino mass. From eq. (3.12) one obtains for the scalar mass parameter

$$
m_{0} \simeq \sqrt{\ell}_{6}\left(\frac{M_{6}}{\Lambda}\right)^{2} \frac{F_{X}}{M_{4}} .
$$

Together with $m_{3 / 2}=F_{X} /\left(\sqrt{3} M_{4}\right)$ this yields

$$
m_{3 / 2} \simeq \frac{1}{\sqrt{3 \ell_{6}}}\left(\frac{\Lambda}{M_{6}}\right)^{2} m_{0} \simeq 0.01\left(\frac{\Lambda}{M_{6}}\right)^{2} m_{0} .
$$

Hence, unless the cutoff significantly exceeds the $6 \mathrm{~d}$ Planck mass, the gravitino will be the lightest superparticle. The result (3.19) is consistent with the analysis carried out in [49]. ${ }^{8}$

Let us finally consider the first and second quark-lepton generations, which are localized at two equivalent fixed points (see [43]) that may or may not coincide with the localization of the supersymmetry breaking fields. In the second case one has

$$
-\mathcal{L}_{\text {soft }}^{\prime}=\tilde{m}_{0}^{2} \sum_{i=1,2}\left(\left|Q_{i}\right|^{2}+\left|U_{i}\right|^{2}+\left|D_{i}\right|^{2}+\left|L_{i}\right|^{2}+\left|E_{i}\right|^{2}\right),
$$

with $\tilde{m}_{0}=0$, correponding to the boudary conditions of gaugino mediation. ${ }^{9}$ In the first case, the scalar mass terms are obtained from eq. (3.7),

$$
-\mathcal{L}_{s b}^{\prime}=\frac{\Lambda^{4}}{\epsilon \ell_{4}} \sum_{i=1,2} \int \frac{d^{4} \theta}{\Lambda^{2}} \frac{|\widehat{X}|^{2}}{\Lambda^{2}}\left(\frac{\left|\widehat{Q}_{i}\right|^{2}}{\Lambda^{2}}+\frac{\left|\widehat{U}_{i}\right|^{2}}{\Lambda^{2}}+\frac{\left|\widehat{D}_{i}\right|^{2}}{\Lambda^{2}}+\frac{\left|\widehat{L}_{i}\right|^{2}}{\Lambda^{2}}+\frac{\left|\widehat{E}_{i}\right|^{2}}{\Lambda^{2}}\right) .
$$

Performing the transition to canonically normalized fields using (3.4), one finds in the case of strong coupling $(\epsilon=1)$,

$$
\tilde{m}_{0} \simeq \frac{1}{\kappa} m_{0} \sim 4 m_{0} .
$$

Hence, in this case, unlike gaugino mediation, first- and second-generation scalars will be heavier than third-generation scalars.

\footnotetext{
${ }^{8}$ Note that the predictions of masses obtained from naive dimensional analysis have an uncertainty of $\mathcal{O}(1)$. This includes the effect of a colour factor which was included in the calculations in [49] and which has been omitted in the present discussion for simplicity.

${ }^{9}$ Strictly speaking this is not possible with the exact particle content of [43], since in this model there is no suitable brane-localized singlet which could play the role of $X$. However a slight variation of this model might well contain a suitable candidate.
} 


\section{Prospects for phenomenology and outlook}

In the six-dimensional GUT model which we discussed in the previous section, the localization of fields and the breaking of supersymmetry by a brane field determine the pattern of scalar and gaugino masses. The Higgs bosons, third generation squarks and sleptons, and gauginos are bulk fields. Their masses depend on $\kappa=M_{1 / 2} / m_{0}$, as determined by the matching scale $M_{S}$, the sign of $\widehat{A}_{t}$ and $m_{0}$ (which in turn is related to the matching scale by renormalization group running). In figure 2 the gluino mass at $M_{S}$ is shown as function of $M_{S}$ for both signs of $\widehat{A}_{t}$. The resulting predicted range of gluino masses,

$$
\left.2 \mathrm{TeV} \lesssim M_{3}\right|_{M_{S}} \lesssim 5 \mathrm{TeV}
$$

is a consequence of the allowed range of matching scales and the sign ambiguity of $\widehat{A}_{t}$.

The first two generations are brane fields. Their masses strongly depend on the localization of the supersymmetry breaking field $X$. There are two possibilities:

(A) The matter fields and $X$ are localized on different branes. This implies the familiar pattern of gaugino mediation, and squarks and sleptons of the first two generations are lighter than those of the third generation. ${ }^{10}$

(B) The matter fields and $X$ are localized on the same brane. According to eq. (3.22), derived in the previous section, the squarks and sleptons of the first two generations are then heavier than those of the third generation.

A further important parameter is the higgsino mass $\mu$. If $\mu$ is generated independently of supersymmetry breaking, generically one would expect $\mu \sim M_{S} / \tan \beta$, as discussed in section 2. In the model of section 3 , since $B \mu$ is enhanced by a factor $1 / \kappa$, we estimate $\mu \sim \kappa M_{S} / \tan \beta$, which implies that for $M_{S}=5 \pm 1.5 \mathrm{TeV}$ and moderately large $\tan \beta$, the $\mu$ parameter should actually be close to the electroweak scale $(|\mu| \lesssim 100 \mathrm{GeV}$ being excluded by chargino searches). A soft upper bound can be estimated by conservatively setting $\tan \beta=5, \kappa=1 / 3$ and $M_{S}=6.5 \mathrm{TeV}$, which yields $\mu \lesssim 450 \mathrm{GeV}$.

In summary, the mass spectrum we predict is characterized by heavy third-generation squarks and sleptons, heavy extra Higgs bosons, gluino masses starting from about $2 \mathrm{TeV}$, higgsino-like charginos and neutralinos with electroweak-scale masses, and squarks and sleptons which are either extremely heavy (B) or generated by gaugino mediation (A). In the latter case, standard SUSY searches for jets and missing energy, as well as searches for direct slepton production, will be promising channels at LHC-14. In any case, the light higgsinos can be searched for and measured at a linear collider [54-56].

Table 1 shows a number of superpartner mass spectra. The first three columns correspond to three different values of $M_{S}$ in scenario (A). For a relatively low matching scale $M_{S}=3.5 \mathrm{TeV}$, gluinos and squarks should be found during the next LHC run, and sleptons should also be easy to see as the slepton masses are already at the border of the present exclusion bounds $[57,58]$. The case of an intermediate matching scale $M_{S}=5 \mathrm{TeV}$ is more

\footnotetext{
${ }^{10}$ Note that for the same localization of fields, but a different mechanism of supersymmetry breaking, third generation squarks and sleptons can also be lighter than those of the first two generation [52, 53].
} 


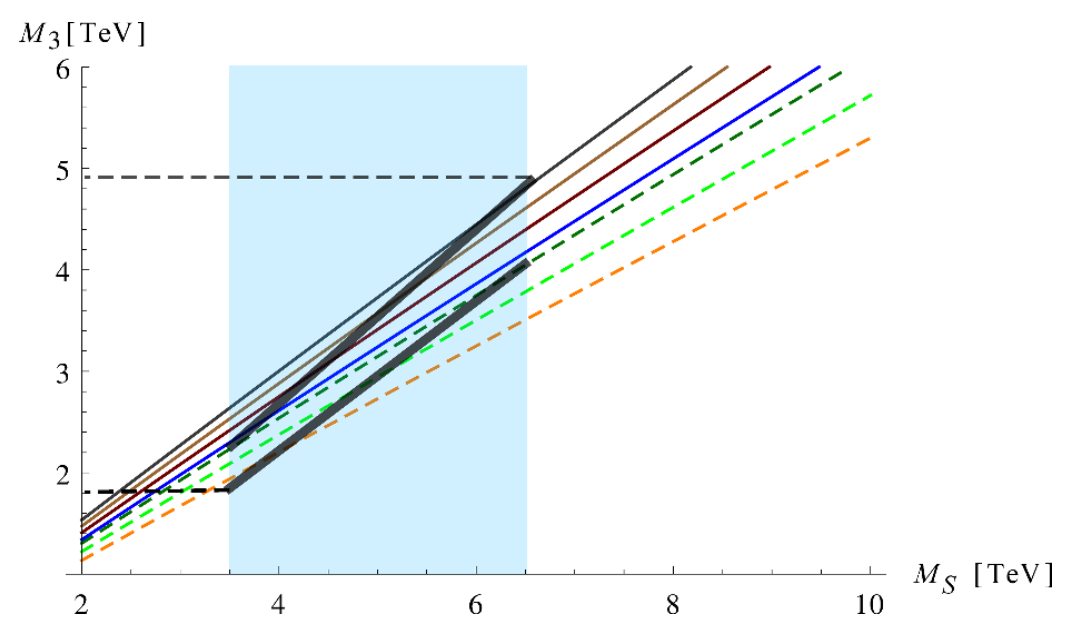

Figure 2. The running gluino mass $\left.M_{3}\right|_{M_{S}}$ as a function of the matching scale $M_{S}$ for various values of the parameter $\kappa=M_{1 / 2} / m_{0}$. Top to bottom curves: $\kappa$ between 0.33 and 0.19 in steps of 0.02 . The solid lines correspond to $\widehat{A}_{t}=+M_{1 / 2}$ and the dashed lines to $\widehat{A}_{t}=-M_{1 / 2}$, with the colour coding the same as in figure 1. Note that the relation between $M_{S}$ and $M_{3}$ for fixed $\kappa$ is only approximately linear. As before, $\tan \beta=15, M_{\mathrm{GUT}}=1.5 \times 10^{16} \mathrm{GeV}$. We have indicated the range of $M_{S}$ preferred by the Higgs mass (which we took to be $5 \pm 1.5 \mathrm{TeV}$ ). We have also indicated the predictions for $M_{3}$ as a function of $M_{S}$, for the two cases $\widehat{A}_{t}= \pm M_{1 / 2}$ (black strips), and the minimal and maximal $M_{3}$ which can be obtained (dashed horizontal lines), when restricting $\left|m_{H_{u}}\right|$ to be of the order of the electroweak scale as in figure 1 . One finds $1.8 \mathrm{TeV} \lesssim M_{3} \lesssim 4.9 \mathrm{TeV}$.

challenging, but squarks and gluinos may still be accessible at high integrated luminosities. The third case of $M_{S}=6.5 \mathrm{TeV}$ places squarks and gluinos out of LHC reach.

The last column of table 1 shows a spectrum for the case that the first- and secondgeneration scalar masses are non-vanishing at the GUT scale and given by $M_{\text {soft }}=30 \mathrm{TeV}$ (scenario (B) above). In this case the overall soft mass scale also for the third generation and the gluinos is higher. The reason is that we are keeping $M_{S}=5 \mathrm{TeV}$ fixed, and the first two squark generations significantly decrease the stop masses when running down from the GUT scale through two-loop effects, up to a point where the stop mixing contribution to the lightest Higgs mass can become very significant [54, 59-61]. This case is not covered by our semi-analytic discussion in section 2, which does not account for possible large contributions to the running from the first two generations, but can nevertheless be dealt with numerically. As is evident from table 1, all states are too heavy to be seen at colliders in the foreseeable future, with the possible exception of the higgsinos.

Finally, the matching scale $M_{S}$ also determines the gravitino mass. From eq. (3.19) one obtains

$$
40 \mathrm{GeV} \simeq m_{3 / 2} \simeq 80 \mathrm{GeV} .
$$

Here we have chosen the $6 \mathrm{~d}$ Planck mass as the cutoff scale, and we have varied $m_{0}$ between $4 \mathrm{TeV}$ and $8 \mathrm{TeV}$ according to table 1 .

The starting point of our discussion has been the compatibility of the measured Higgs boson mass, and the associated large matching scale $M_{S}$, with a Fermi scale significantly 


\begin{tabular}{|ccccc|}
\hline \multicolumn{4}{c}{ light 1st \& 2nd generation } & heavy 1st \& 2nd generation \\
\hline$\chi_{1}^{0}$ & 127 & 109 & 141 & $M_{S}=5 \mathrm{TeV}$ \\
$\chi_{2}^{0}$ & 140 & 116 & 146 & 185 \\
$\chi_{1}^{ \pm}$ & 133 & 112 & 144 & 189 \\
$\chi_{3}^{0}$ & 430 & 700 & 990 & 187 \\
$\chi_{4}^{0}, \chi_{2}^{ \pm}$ & 820 & 1300 & 1900 & 1100 \\
$H_{0}, A_{0}, H^{ \pm}$ & 4200 & 5900 & 7500 & 2100 \\
$\tilde{g}$ & 2200 & 3500 & 4800 & 7200 \\
$\tilde{u}_{i}, \tilde{d}_{i}, \tilde{c}_{i}, \tilde{s}_{i}$ & $1800-2000$ & $2800-3000$ & $3900-4100$ & 5600 \\
$\tilde{t}_{1}$ & 3100 & 4500 & 5800 & $3 \times 10^{4}$ \\
$\tilde{t}_{2}$ & 4000 & 5600 & 7300 & 4400 \\
$\tilde{b}_{1}$ & 4000 & 5700 & 7400 & 5900 \\
$\tilde{b}_{2}$ & 4600 & 6500 & 8400 & 6000 \\
$\tilde{\mu}_{1}, \tilde{e}_{1}$ & 350 & 560 & 800 & 7400 \\
$\tilde{\mu}_{2}, \tilde{e}_{2}$ & 610 & 1000 & 1400 & $3 \times 10^{4}$ \\
$\tilde{\tau}_{1}$ & 4300 & 5900 & 7500 & $3 \times 10^{4}$ \\
$\tilde{\tau}_{2}$ & 4400 & 6000 & 7700 & 7400 \\
\hline
\end{tabular}

Table 1. Example mass spectra computed with SOFTSUSY 3.3.10 [32] for different matching scales. The parameters are $\tan \beta=15, \widehat{A}_{t, b}=0, M_{1 / 2}=(1,1.6,2.25,2.45) \mathrm{TeV}, m_{0}=$ $(4.35,6.0,7.6,7.7) \mathrm{TeV}$ for the third generation and the Higgs fields. For the three columns on the left, the GUT-scale scalar soft masses of the first two generations vanish, whereas for the rightmost column they are $\tilde{m}_{0}=30 \mathrm{TeV}$. All masses in the table are in units of $\mathrm{GeV}$.

smaller than $M_{S}$. We have shown that for a small higgsino mass $\mu$, not controlled by supersymmetry breaking, and universal Higgs and stop masses at the GUT scale, a small Fermi scale arises for suitable relations between gaugino and scalar masses. It is interesting that a simple example can be obtained within the context of a higher-dimensional GUT model. The matching scale, together with the related gaugino-scalar mass ratio, and the value of $\tan \beta$ determine the superparticle mass spectrum. If the matching scale turns out to be lower than about $5 \mathrm{TeV}$, this scenario will be probed by the upcoming next LHC run, with searches for gluinos, squarks and also sleptons being promising channels. Moreover, our setup favours light higgsinos, which can be searched for at a linear collider. The lightest superparticle is the gravitino.

\section{Acknowledgments}

We thank J. Kersten, M. Ratz und M. Winkler for helpful comments. This work was supported in part by the German Science Foundation (DFG) within the Collaborative Research Center 676 "Particles, Strings and the Early Universe". The work of FB was supported in part by ERC Advanced Grant 267985 "Electroweak Symmetry Breaking, Flavour and Dark Matter".

Open Access. This article is distributed under the terms of the Creative Commons Attribution License (CC-BY 4.0), which permits any use, distribution and reproduction in any medium, provided the original author(s) and source are credited. 


\section{References}

[1] S. Dimopoulos and H. Georgi, Softly broken supersymmetry and SU(5), Nucl. Phys. B 193 (1981) 150 [INSPIRE].

[2] L.E. Ibáñez and G.G. Ross, Low-energy predictions in supersymmetric grand unified theories, Phys. Lett. B 105 (1981) 439 [INSPIRE].

[3] S. Dimopoulos, S. Raby and F. Wilczek, Supersymmetry and the scale of unification, Phys. Rev. D 24 (1981) 1681 [INSPIRE].

[4] ATLAS collaboration, Search for new phenomena in final states with large jet multiplicities and missing transverse momentum at $\sqrt{s}=8$ TeV proton-proton collisions using the ATLAS experiment, JHEP 10 (2013) 130 [arXiv: 1308.1841] [INSPIRE].

[5] ATLAS collaboration, Search for strongly produced supersymmetric particles in decays with two leptons at $\sqrt{s}=8 \mathrm{Te}$, ATLAS-CONF-2013-089 (2013).

[6] ATLAS collaboration, Search for squarks and gluinos in events with isolated leptons, jets and missing transverse momentum at $\sqrt{s}=8$ TeV with the ATLAS detector,

ATLAS-CONF-2013-062 (2013).

[7] ATLAS collaboration, Search for squarks and gluinos with the ATLAS detector in final states with jets and missing transverse momentum and $20.3 \mathrm{fb}^{-1}$ of $\sqrt{\mathrm{s}}=8$ TeV proton-proton collision data, ATLAS-CONF-2013-047 (2013).

[8] CMS collaboration, Search for supersymmetry in hadronic final states with missing transverse energy using the variables $\alpha_{T}$ and b-quark multiplicity in pp collisions at $8 \mathrm{TeV}$, Eur. Phys. J. C 73 (2013) 2568 [arXiv:1303.2985] [InSPIRE].

[9] CMS collaboration, Search for new physics in events with same-sign dileptons and jets in pp collisions at $8 \mathrm{TeV}$, CMS-PAS-SUS-13-013 (2013).

[10] CMS collaboration, Search for new physics in the multijets and missing momentum final state in proton-proton collisions at 8 TeV, CMS-PAS-SUS-13-012 (2013).

[11] CMS collaboration, Search for supersymmetry using razor variables in events with b-jets in pp collisions at 8 TeV, CMS-PAS-SUS-13-004 (2013).

[12] ATLAS collaboration, Observation of a new particle in the search for the standard model Higgs boson with the ATLAS detector at the LHC, Phys. Lett. B 716 (2012) 1 [arXiv: 1207.7214] [INSPIRE].

[13] CMS collaboration, Observation of a new boson at a mass of $125 \mathrm{GeV}$ with the CMS experiment at the LHC, Phys. Lett. B 716 (2012) 30 [arXiv:1207.7235] [INSPIRE].

[14] CMS collaboration, Observation of a new boson with mass near 125 GeV in pp collisions at $\sqrt{s}=7$ and $8 \mathrm{TeV}$, JHEP 06 (2013) 081 [arXiv: 1303.4571] [INSPIRE].

[15] N. Arkani-Hamed and S. Dimopoulos, Supersymmetric unification without low energy supersymmetry and signatures for fine-tuning at the LHC, JHEP 06 (2005) 073 [hep-th/0405159] [INSPIRE].

[16] G. Giudice and A. Romanino, Split supersymmetry, Nucl. Phys. B 699 (2004) 65 [Erratum ibid. B 706 (2005) 65-89] [hep-ph/0406088] [INSPIRE].

[17] K.L. Chan, U. Chattopadhyay and P. Nath, Naturalness, weak scale supersymmetry and the prospect for the observation of supersymmetry at the Tevatron and at the CERN LHC, Phys. Rev. D 58 (1998) 096004 [hep-ph/9710473] [INSPIRE]. 
[18] J.L. Feng, K.T. Matchev and T. Moroi, Multi-TeV scalars are natural in minimal supergravity, Phys. Rev. Lett. 84 (2000) 2322 [hep-ph/9908309] [INSPIRE].

[19] J.L. Feng, K.T. Matchev and T. Moroi, Focus points and naturalness in supersymmetry, Phys. Rev. D 61 (2000) 075005 [hep-ph/9909334] [INSPIRE].

[20] J.L. Feng, K.T. Matchev and D. Sanford, Focus point supersymmetry redux, Phys. Rev. D 85 (2012) 075007 [arXiv: 1112.3021] [INSPIRE].

[21] H. Abe, T. Kobayashi and Y. Omura, Relaxed fine-tuning in models with non-universal gaugino masses, Phys. Rev. D 76 (2007) 015002 [hep-ph/0703044] [INSPIRE].

[22] D. Horton and G.G. Ross, Naturalness and focus points with non-universal gaugino masses, Nucl. Phys. B 830 (2010) 221 [arXiv:0908.0857] [INSPIRE].

[23] F. Brümmer and W. Buchmüller, Light higgsinos as heralds of higher-dimensional unification, JHEP 07 (2011) 010 [arXiv:1105.0802] [INSPIRE].

[24] F. Brummer and W. Buchmüller, The Fermi scale as a focus point of high-scale gauge mediation, JHEP 05 (2012) 006 [arXiv:1201.4338] [INSPIRE].

[25] J.E. Younkin and S.P. Martin, Non-universal gaugino masses, the supersymmetric little hierarchy problem and dark matter, Phys. Rev. D 85 (2012) 055028 [arXiv:1201.2989] [INSPIRE].

[26] T.T. Yanagida and N. Yokozaki, Focus point in gaugino mediation - Reconsideration of the fine-tuning problem, Phys. Lett. B $\mathbf{7 2 2}$ (2013) 355 [arXiv:1301.1137] [INSPIRE].

[27] F. Brümmer, M. Ibe and T.T. Yanagida, Focus point gauge mediation in product group unification, Phys. Lett. B 726 (2013) 364 [arXiv:1303.1622] [INSPIRE].

[28] U. Ellwanger, C. Hugonie and A.M. Teixeira, The next-to-minimal supersymmetric standard model, Phys. Rept. 496 (2010) 1 [arXiv:0910.1785] [InSPIRE].

[29] P. Batra, A. Delgado, D.E. Kaplan and T.M. Tait, The Higgs mass bound in gauge extensions of the minimal supersymmetric standard model, JHEP 02 (2004) 043 [hep-ph/0309149] [INSPIRE].

[30] N. Craig, The state of supersymmetry after run I of the LHC, arXiv:1309.0528 [INSPIRE].

[31] S.P. Martin, A supersymmetry primer, in Perspectives on supersymmetry II, G.L. Kane ed., World Scientific, Singapore (2010), hep-ph/9709356 [INSPIRE].

[32] B.C. Allanach, SOFTSUSY: a program for calculating supersymmetric spectra, Comput. Phys. Commun. 143 (2002) 305 [hep-ph/0104145] [INSPIRE].

[33] A. Djouadi, J.-L. Kneur and G. Moultaka, SuSpect: a Fortran code for the supersymmetric and Higgs particle spectrum in the MSSM, Comput. Phys. Commun. 176 (2007) 426 [hep-ph/0211331] [INSPIRE].

[34] S. Heinemeyer, W. Hollik and G. Weiglein, FeynHiggs: a program for the calculation of the masses of the neutral CP even Higgs bosons in the MSSM, Comput. Phys. Commun. 124 (2000) 76 [hep-ph/9812320] [INSPIRE].

[35] S. Heinemeyer, W. Hollik and G. Weiglein, The masses of the neutral CP-even Higgs bosons in the MSSM: accurate analysis at the two loop level, Eur. Phys. J. C 9 (1999) 343 [hep-ph/9812472] [INSPIRE]. 
[36] G. Degrassi, S. Heinemeyer, W. Hollik, P. Slavich and G. Weiglein, Towards high precision predictions for the MSSM Higgs sector, Eur. Phys. J. C 28 (2003) 133 [hep-ph/0212020] [INSPIRE].

[37] M. Frank et al., The Higgs boson masses and mixings of the complex MSSM in the Feynman-diagrammatic approach, JHEP 02 (2007) 047 [hep-ph/0611326] [INSPIRE].

[38] J.L. Feng, P. Kant, S. Profumo and D. Sanford, Three-loop corrections to the Higgs boson mass and implications for supersymmetry at the LHC, Phys. Rev. Lett. 111 (2013) 131802 [arXiv:1306.2318] [INSPIRE].

[39] P. Kant, R. Harlander, L. Mihaila and M. Steinhauser, Light MSSM Higgs boson mass to three-loop accuracy, JHEP 08 (2010) 104 [arXiv: 1005.5709] [INSPIRE].

[40] D.M. Pierce, J.A. Bagger, K.T. Matchev and R.-j. Zhang, Precision corrections in the minimal supersymmetric standard model, Nucl. Phys. B 491 (1997) 3 [hep-ph/9606211] [INSPIRE].

[41] C. Csáki, A. Falkowski, Y. Nomura and T. Volansky, New approach to the $\mu$-B $\mu$ problem of gauge-mediated supersymmetry breaking, Phys. Rev. Lett. 102 (2009) 111801 [arXiv: 0809.4492] [INSPIRE].

[42] A. De Simone, R. Franceschini, G.F. Giudice, D. Pappadopulo and R. Rattazzi, Lopsided gauge mediation, JHEP 05 (2011) 112 [arXiv:1103.6033] [INSPIRE].

[43] W. Buchmüller, C. Lüdeling and J. Schmidt, Local SU(5) unification from the heterotic string, JHEP 09 (2007) 113 [arXiv:0707.1651] [INSPIRE].

[44] W. Buchmüller, K. Hamaguchi, O. Lebedev and M. Ratz, Supersymmetric standard model from the heterotic string, Phys. Rev. Lett. 96 (2006) 121602 [hep-ph/0511035] [InSPIRE].

[45] O. Lebedev et al., A mini-landscape of exact MSSM spectra in heterotic orbifolds, Phys. Lett. B 645 (2007) 88 [hep-th/0611095] [INSPIRE].

[46] P. Hosteins, R. Kappl, M. Ratz and K. Schmidt-Hoberg, Gauge-top unification, JHEP 07 (2009) 029 [arXiv: 0905.3323] [INSPIRE].

[47] H.M. Lee, H.P. Nilles and M. Zucker, Spontaneous localization of bulk fields: the six-dimensional case, Nucl. Phys. B 680 (2004) 177 [hep-th/0309195] [InSPIRE].

[48] W. Buchmüller and J. Schmidt, Higgs versus matter in the heterotic landscape, Nucl. Phys. B 807 (2009) 265 [arXiv:0807.1046] [INSPIRE].

[49] W. Buchmüller, K. Hamaguchi and J. Kersten, The gravitino in gaugino mediation, Phys. Lett. B 632 (2006) 366 [hep-ph/0506105] [INSPIRE].

[50] N. Arkani-Hamed, G.F. Giudice, M.A. Luty and R. Rattazzi, Supersymmetry breaking loops from analytic continuation into superspace, Phys. Rev. D 58 (1998) 115005 [hep-ph/9803290] [INSPIRE].

[51] Z. Chacko, M.A. Luty, A.E. Nelson and E. Ponton, Gaugino mediated supersymmetry breaking, JHEP 01 (2000) 003 [hep-ph/9911323] [INSPIRE].

[52] S. Krippendorf, H.P. Nilles, M. Ratz and M.W. Winkler, The heterotic string yields natural supersymmetry, Phys. Lett. B 712 (2012) 87 [arXiv:1201.4857] [INSPIRE].

[53] M. Badziak, S. Krippendorf, H.P. Nilles and M.W. Winkler, The heterotic minilandscape and the $126 \mathrm{GeV}$ Higgs boson, JHEP 03 (2013) 094 [arXiv:1212.0854] [INSPIRE]. 
[54] H. Baer, V. Barger, P. Huang and X. Tata, Natural supersymmetry: LHC, dark matter and ILC searches, JHEP 05 (2012) 109 [arXiv: 1203.5539] [INSPIRE].

[55] H. Baer, V. Barger and P. Huang, Hidden SUSY at the LHC: the light higgsino-world scenario and the role of a lepton collider, JHEP 11 (2011) 031 [arXiv:1107.5581] [INSPIRE].

[56] M. Berggren et al., Tackling light higgsinos at the ILC, Eur. Phys. J. C 73 (2013) 2660 [arXiv: 1307.3566] [INSPIRE].

[57] ATLAS collaboration, Search for direct-slepton and direct-chargino production in final states with two opposite-sign leptons, missing transverse momentum and no jets in 20/fb of pp collisions at $\sqrt{s}=8 \mathrm{TeV}$ with the ATLAS detector, ATLAS-CONF-2013-049 (2013).

[58] CMS collaboration, Search for electroweak production of charginos, neutralinos and sleptons using leptonic final states in pp collisions at 8 TeV, CMS-PAS-SUS-13-006 (2013).

[59] N. Arkani-Hamed and H. Murayama, Can the supersymmetric flavor problem decouple?, Phys. Rev. D 56 (1997) 6733 [hep-ph/9703259] [INSPIRE].

[60] F. Brummer, S. Kraml and S. Kulkarni, Anatomy of maximal stop mixing in the MSSM, JHEP 08 (2012) 089 [arXiv: 1204.5977] [INSPIRE].

[61] M. Badziak, E. Dudas, M. Olechowski and S. Pokorski, Inverted sfermion mass hierarchy and the Higgs boson mass in the MSSM, JHEP 07 (2012) 155 [arXiv:1205.1675] [INSPIRE]. 